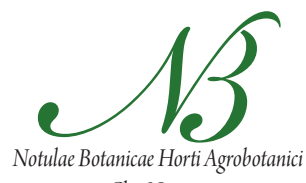

Cluj-Napoca

\title{
Influence of Bulk and Nanoparticles Titanium Foliar Application on some Agronomic Traits, Seed Gluten and Starch Contents of Wheat Subjected to Water Deficit Stress
}

\author{
Amir JABERZADEH', Payam MOAVENI², Hamid Reza \\ TOHIDI MOGHADAM ${ }^{1 *}$, Hossein ZAHEDI $^{3}$ \\ ${ }^{1}$ Department of Agronomy, Varamin-Pishva Branch, Islamic Azad University, Varamin, Iran \\ ${ }^{2}$ Department of Agronomy, Shahr-e-Qods Branch, Islamic Azad University, Tehran, Iran \\ ${ }^{3}$ Department of Agronomy and Plant Breeding, Eslamshahr Branch, Islamic Azad University, \\ Tehran, Iran; hamid_tohidi2008@yahoo.com ("corresponding author)
}

\begin{abstract}
Titanium $(\mathrm{Ti})$ is a very interesting chemical element, especially physiologically. Although $\mathrm{Ti}$ is not toxic for animals and humans, its effects on plants show remarkable concentration dependence. Whereas for plants, it shows beneficial effects on various physiological parameters at low doses. This study was conducted to evaluate the effect of bulk and nanoparticles titanium foliar application on some agronomic traits, seed gluten and starch contents of wheat under water deficit stress conditions during 2010-2011 growing seasons. The experimental design was randomized in complete blocks arranged in split-split plots with four replications. The factors included normal irrigation, water deficit stress (irrigation withholding at two growing stages of stem elongation and flowering), two growing stages for water deficit stress induction and titanium applications, five titanium concentrations, sources including control of titanium oxide (bulk), and three concentrations of $0.01 \%, 0.02 \%$, and $0.03 \%$ of titanium dioxide nanoparticles. Plant height, ear weight, ear number, seed number, 1000-seed weight, final yield, biomass, harvest index, gluten, and starch contents were assayed. The results showed that water deficit stress caused significant decrease in plant growth, yield and yield components. In addition, among the different titanium treatments, titanium dioxide nanoparticles at $0.02 \%$ increased almost all agronomic traits including gluten and starch content. Thus, the application of titanium dioxide nanoparticles under conditions of water deficit stress is recommended.
\end{abstract}

Keywords: gluten, starch, titanium dioxide, wheat, yield and yield components

\section{Introduction}

Titanium is a chemical element with the symbol $\mathrm{Ti}$ and the atomic number 22. It has a low density and is a strong, lustrous, corrosion-resistant transition metal with a silver colour. Titanium has significant biological effects on plants, being beneficial at low levels but toxic at higher concentrations. Although titanium is not toxic for animals and humans, its effects on plants and bacteria show noteworthy concentration dependence. Whereas for bacteria it acts as an antibiotic (Yaghoubi et al., 2000), for plants, it shows beneficial effects on various physiological parameters at low doses [e.g. biomass yield (Pais, 1993), essential element contents (Giménez et al., 1990), chlorophyll contents (Carvajal et al., 1994)] and is toxic at higher levels [chlorosis, retardation of growth (Hruby et al., 2002)]. Although titanium is beneficial, it is not essential; titanium deficiency does not exist. Beneficial effects are often exploited by adding titanium to various complex micronutrient fertilizers. Many investigators (Dumon and Ernst, 1988) demonstrated the promotion of growth by titanium, whether applied as a fertilizer to the soil, or as a spray to the leaves. Pais (1983) carried out numerous field experiments and found that titanium-chelate-treated apple trees yielded the fruits with higher soluble solids, sugar and acid content. The positive effects of titanium treatment were found on rape plant development (an increase of chlorophyll content and photosynthesis intensity), the yield and mass of a thousand seeds of winter wheat, and the yield and sugar content in sugar beets (Grenda, 2003). The most important effects of titanium on plants are enhancement of the yield, an improvement of some essential element contents in plant tissues, and an enhancement of the chlorophyll content in paprika (Capsicum anuum L.) (Hruby et al., 2002). Plants interact with their atmospheric and edaphic environments strongly and are expected to be affected by exposure to engineered nanoparticles (Ruffini Castiglione and Cremonini, 2009). Lu et al. (2002) have shown that a combination of nano-sized $\mathrm{SiO}_{2}$ and $\mathrm{TiO}_{2}$ could increase the nitrate reductase enzyme in soybean (Glycine max L.), increase its abilities of absorbing and utilizing water and fertilizer, promote its antioxidant system, 
202

and, in fact, accelerate its germination and growth. Also, it has been found that $\mathrm{TiO}_{2}$ nanoparticles encourage spinach (Spinacia oleracea) seed germination and plant growth (Zheng et al., 2005). Navarro et al. (2008) stated that engineered nanoparticles could sequester nutrients on their surfaces, and thus, serve as a nutrient stock to the organisms, particularly those engineered nanoparticles having high specific surface area.

Whereas, in the literature there is little evidence on the effect of titanium on crop plants, we have studied the influence of titanium dioxide nanoparticles and common titanium oxide (bulk) on growth, yield and yield components, and also on gluten and starch of wheat under conditions of water deficit stress. The objective of this study, therefore, is to evaluate the response of wheat plant to titanium and water deficit stress.

\section{Materials and methods}

The field experiment was conducted at Shahriar, Iran $\left(35^{\circ} 39^{\prime} 52^{\prime \prime} \mathrm{N}, 51^{\circ} 03^{\prime} 33^{\prime \prime} \mathrm{E}\right)$ during the 2010-2011 growing season. The yearly average precipitation (30 years longterm period) was $244 \mathrm{~mm}$, mostly concentrated during the autumn and winter months (November to February). The experimental design was randomized complete blocks arranged in spilt-split plot with four replications. Irrigation was the main factor at two levels, normal irrigation, water deficit stress (irrigation withholding at two growing stages of stem elongation and flowering). Plant growth stages were considered as sub-factors and, finally, different doses of titanium (Control, $0.01 \%, 0.02 \%, 0.03 \%$ titanium nanoparticle and bulk titanium) were allocated to sub-sub plots. After plowing and disks in autumn, soil samples were collected to determine the soil characteristics. The soil had clay loam texture containing $28.6 \%$ sand, $25.2 \%$ silt, and $46.2 \%$ clay. The soil $\mathrm{pH}$ and EC were measured as 7.7 and $3.4 \mathrm{~mm}$ hos $\mathrm{cm}^{-1}$, respectively. When plots were prepared there were 80 plots with $2 \mathrm{~m}$ width and $4 \mathrm{~m}$ length. There was a $2-\mathrm{m}$ alley between plots and blocks to avoid lateral water movement. Before seed sowing, $150 \mathrm{~kg}$ ha ${ }^{-1}$ super phosphate triple was mixed into the soil. Wheat seeds (Triticum aestivum L. c.v 'Pishtaz') were sown by hand on $15^{\text {th }}$ November at a depth of $3 \mathrm{~cm}$. The planting density was 350 individuals per square meter. Irrigation was performed as furrow irrigation immediately. Second irrigation was done 3 days after the first one to get the best seed germination and seedling establishment. Weeds were controlled manually during the growing season. Water deficit stress treatment was induced by irrigation withholding in spring at two different stages, stem elongation and flowering. It is worth mentioning that nitrogen fertilizer (300 kg ha ${ }^{-1}$ urea) was applied at the tillering and booting stages. In order to prepare titanium dioxide nanoparticles, $20 \mathrm{~g}$ titanium dioxide was dissolved into water and then
$0.01 \mathrm{ml}$ of solution was filled up to $1000 \mathrm{ml}$. Thus, different concentrations of titanium dioxide $(0.01 \%, 0.02 \%$, and $0.03 \%$ ) were prepared. An ultrasound instrument was used to homogenize the solution. Bulk titanium dioxide was purchased from Advanced Material Company (United States). To make bulk solution, $6 \mathrm{~g}$ solid of titanium was dissolved in $100 \mathrm{ml}$ distilled water, then $1 \mathrm{ml}$ of solution was filled up to $1000 \mathrm{ml}$. Titanium dioxide nanoparticles were sprayed on plants using a calibrated pressurized backpack sprayer (capacity $20 \mathrm{l}$ ) at the stem elongation and flowering stages at the end of water deficit stress induction. Plants were treated with $240 \mathrm{ml}$ titanium solution per square meter. Control plants were treated with distilled water. At the end of the growing season, crop was harvested and the following traits were investigated: Plant height, ear weight per square meter, ear number per square meter, seed number per square meter, 1000 seed weight, final yield, biomass, and harvest index. Gluten and starch were measured according to the methods of Graveland and Henderson (2000) and Fulai et al. (2004), respectively. In order to purify gluten, $50 \mathrm{~g}$ of wheat flour was washed by chloroform to de-lipidation and then dissolved in $600 \mathrm{ml}$ distilled water. The mixture was centrifuged at $1500 \mathrm{xg}$ for $10 \mathrm{~min}$; sediment was dissolved in $0.5 \mathrm{M}$ acetic acid. After adding $600 \mathrm{ml}$ distilled water, $\mathrm{pH}$ was adjusted on 3.9 . After $1 \mathrm{~h}$, the samples were centrifuged at $500 \mathrm{xg}$ again for $10 \mathrm{~min}$. Sediment was dissolved in acetic acid and used for electrophoresis procedures. In brief, after preparing SDSPAGE, the extracted gluten was dissolved in Tris buffer, then loaded and run onto the gel. After about $100 \mathrm{~min}$, the bands were fixed by trichloroacetic acid solution. After dying the gluten and standard bands, gluten concentration was estimated using a specific scanner. Starch content was estimated based on spectrophotometry. Thus, $50 \mathrm{~g}$ of wheat flour was washed by ethanol $(80 \%)$ and, after boiling for $15 \mathrm{~min}$, the samples were centrifuged at 20,000xg for $10 \mathrm{~min}$. Sediment was dissolved in dichloromethane and centrifuged again at $4000 \mathrm{xg}$ for $10 \mathrm{~min}$. Supernatant was removed gently and dried at $45^{\circ} \mathrm{C}$. What remained was dissolved in distilled water and was passed from the hydroxyl column in order to separate the sugars. Remained starch was dissolved in sodium phosphate buffer and digested by digestive solution containing $100 \mathrm{ml}$ of $15 \mathrm{mM}$ $\mathrm{MgCl}_{2}$ and 2.5 unit amyloglucosidase enzyme for $1 \mathrm{~h}$ at $55^{\circ} \mathrm{C}$. Released glucose reacted with the amyloglucosidase enzyme and, after composing with dianisidine solution, the reaction was stopped using sulphuric acid. Absorbance was read at $540 \mathrm{~nm}$ and starch content was calculated according to the standard curve. Analysis of variance were carried out using the GLM procedure (SAS Institute, 2002), assuming that the residuals were random, homogenous and with a normal distribution about a mean of zero. Treatment means were compared using LSMEANS $(p \leq 0.05)$. 


\section{Results and discussion}

The results showed that the individual effect of irrigation, the combined effect of growth stages and titanium concentrations, and the combined effect of irrigation and growth stages had a significant impact on plant height. In addition, the triple combined effect was significant (Tab. 1). Comparison of means demonstrated that water deficit stress considerably decreased plant height (Tab. 2), although there was no significant difference between the two growing stages regarding plant height (Tab. 2). In the case of titanium application, the highest plants were harvested from the control plot, whereas the shortest plants were found in those plots which were treated with titanium dioxide $0.01 \%$ (Tab. 2). When it comes to the combined effects, results show that the highest plants were observed in non-stressed plots. By contrast, when titanium dioxide $(0.01 \%)$ was applied on stressed plants during the flowering stage, the shortest plants were produced (Tab. 3). The results were strongly dependent on water deficit stress and growing stages, but we generalized some trends. The effect of water deficit stress was obvious on all the traits of wheat. Plant height was significantly affected by water stress. Plant height plays an important role in photosynthesis. Malik and Hassan (2002) and Khanzada et al. (2001) have earlier reported that shoot length was significantly reduced under water stress. Similarly, Inamullah et al. (1999) also observed that plant height in wheat varieties reduced significantly under water deficit stress when it was compared with irrigated plants.

The individual effect of titanium concentrations, the combined effect of irrigation and titanium concentrations, and the combined effect of growth stages and titanium concentrations were significant (Tab. 1). As can be seen from Tab. 2, there is no significant difference between complete irrigation and withholding irrigation regarding ear weight per square meter. Furthermore, the two growing stages were not statistically different. The maximum ear weight was observed when titanium dioxide nanoparticles $(0.02 \%)$ were applied on plants; however, there was no significant difference between this treatment and titanium oxide (bulk) treatment (Tab. 2). The maximum and minimum ear weight per square meter were obtained when non-stressed plants were treated with titanium dioxide nanoparticles $(0.02 \%)$ and titanium dioxide nanoparticles $(0.01 \%)$ at the flowering stage, respectively (Tab. $3)$. Ear number per square meter was significantly affected by irrigation withholding and decreased due to water stress. There was no significant difference between two growing stages regarding ear number (Tab. 2). In addition, titanium dioxide nanoparticles at $0.02 \%$ concentration increased ear number (Tab. 2). The combined effects among irrigation, growing stages, and titanium concentrations indicate that the most ears were produced when titanium dioxide nanoparticles $(0.02 \%)$ were applied on non-stressed plants at the stem elongation stage (Tab. 3), whereas the least ears were obtained from stressed plants and no titanium application at the flowering stage (Tab. 3). Qadir et al. (1999) also found that water deficit stress reduced the spikelets per spike in wheat. Tompkins et al. (1991) reported the significant suppressive effect of water deficit stress on the number of seeds per ear. Khanzada et al. (2001) and Qadir et al. (1999) have earlier reported that water deficit stress throughout the vegetative and reproductive development caused a significant reduction in the number of seeds per ear in wheat. The effects of irrigation and titanium concentrations were significant on seed number per square meter. In addition, all combined effects were significant as well (Tab. 1). Water deficit stress led to the decrease in seed number per square meter during both growing stages; however, there was no significant difference between the two growing stages (Tab. 2). Titanium application, whether titanium dioxide nanoparticles or titanium oxide (bulk), increased seed number per square meter compared with control, although titanium dioxide nanoparticles $(0.02 \%)$ produced the highest number of seeds (Tab. 2). When the effects of three experimental factors were combined, the highest seed number was obtained when titanium dioxide nanoparticles $(0.02 \%)$ and titanium oxide (bulk) were applied on non-stressed plants at the stem elongation and flowering stages, respectively (Tab. 3). Water deficit stress and titanium application had significant effects on 1000 seed weight but the effect of the growing stage was not significant (Tab. 1). An interaction among these factors showed a significant effect as well. Irrigation withholding decreased 1000 seed weight at both growing stages, whereas there was no significant difference between the stem elongation and flowering stages (Tab. 2). Application of titanium dioxide nanoparticles increased 1000 seed weight compared to titanium oxide (bulk) or control treatment (Tab. 2). The highest 1000 seed weight was observed when non-stressed plants were treated with titanium dioxide nanoparticles $(0.02 \%)$ at the flowering stage; on the other hand, the lowest 1000 seed weight was obtained from those plants which were not treated with titanium but stressed at the flowering stage (Tab. 3). Analysis of variance showed that all individual and combined effects were significant except for the combined effect of irrigation and growth stage (Tab. 1). Water deficit stress drastically decreased final seed yield. In addition, water deficit stress induction at the flowering stage led to a significant decrease in seed yield compared to stress at the stem elongation stage (Tab. 2). Thus, the flowering stage is more sensitive to water stress. Generally, titanium application caused seed yield increment (Tab. 2), however, titanium dioxide nanoparticles $(0.02 \%)$ application was more effective in improving yield (Tab. 2). The highest seed yield was obtained when non-stressed plants were treated with titanium dioxide nanoparticles $(0.02 \%)$ during the stem elongation stage (Tab. 3).

According to the analysis of variance (Tab. 1), irrigation and titanium application had a significant effect on 
Tab. 1. Analysis of variance on some agronomic traits, gluten and starch of wheat affected by irrigation, growth stages and titanium concentrations

\begin{tabular}{|c|c|c|c|c|c|c|c|c|c|c|c|}
\hline Sources of variation & d.f & Plant height & Ear weight & Ear number & Seed number & $\begin{array}{c}\text { 1000-seed } \\
\text { weight }\end{array}$ & Final yield & Biomass & Harvest index & Gluten & Starch \\
\hline Replication & 3 & $10.3744700^{\mathrm{ns}}$ & $387024.399^{* *}$ & $113146.5458^{* *}$ & $242843788^{* *}$ & $13.3790000^{* *}$ & $11762391.39^{* *}$ & $260232957.0^{* *}$ & $17.2812567^{\mathrm{ns}}$ & $0.034633^{\mathrm{ns}}$ & $1.553792^{\mathrm{ns}}$ \\
\hline Irrigation & 1 & $188.6822450^{* *}$ & $513.439^{\text {ns }}$ & $79191.1125^{* *}$ & $187099726^{* *}$ & $459.8405000^{* *}$ & $5087126.28^{* *}$ & $36896861.3^{* *}$ & $487.9732050^{* *}$ & $244.7900450^{* *}$ & $1485.226125^{* *}$ \\
\hline Error (a) & 3 & 18.9931550 & 46124.142 & 16416.8458 & 33201530 & 2.3968333 & 478053.33 & 3056771.5 & 8.6634083 & 0.1825350 & 0.583792 \\
\hline Growth stage & 1 & $11.5520000^{\mathrm{ns}}$ & $12517.756^{\mathrm{ns}}$ & $437.1125^{\mathrm{ns}}$ & $3950583^{\mathrm{ns}}$ & $0.760500^{\mathrm{ns}}$ & $871593.88^{*}$ & $48285888.8^{\mathrm{ns}}$ & $69.0432800^{* *}$ & $0.0540800^{\mathrm{ns}}$ & $0.210125^{\mathrm{ns}}$ \\
\hline Irrigation $\times$ growth stage & 1 & $61.7058450^{*}$ & $2848.049^{\mathrm{ns}}$ & $49750.3125^{* *}$ & $55244714^{* *}$ & $0.000000^{\mathrm{ns}}$ & $413036.91^{\text {ns }}$ & $131220.0^{\mathrm{ns}}$ & $73.8432450^{* *}$ & $0.0014450^{\mathrm{ns}}$ & $0.351125^{\mathrm{ns}}$ \\
\hline Error (b) & 6 & 34.7121225 & 12038.195 & 8331.9125 & 14375568 & 1.5722500 & 72452.27 & 8162096.3 & 6.1423958 & 0.1177192 & 1.087292 \\
\hline Concentration & 4 & $30.3010456^{\mathrm{ns}}$ & $42413.617^{* *}$ & $22763.9188^{\mathrm{ns}}$ & $70046909^{* *}$ & $1.6265625^{* *}$ & $1412461.84^{* *}$ & $35401656.0^{* *}$ & $108.815863^{* *}$ & $0.9993081^{* *}$ & $9.067813^{* *}$ \\
\hline Irrigation $\times$ concentration & 4 & $47.7314294^{*}$ & $108359.281^{* *}$ & $69908.5813^{* *}$ & $180688827^{* *}$ & $18.6295625^{* *}$ & $2143772.01^{* *}$ & $31397328.5^{* *}$ & $21.0887363^{* *}$ & $2.9593919^{* *}$ & $9.831438^{* *}$ \\
\hline Growth stage $\times$ concentration & 4 & $56.5422969^{* *}$ & $221222.379^{* *}$ & $172586.1438^{* *}$ & $358371140^{* *}$ & $11.4733125^{* *}$ & $5192796.38^{* *}$ & $143408542.1^{* *}$ & $27.2678800^{* *}$ & $0.3335081^{\text {ns }}$ & $1.954188^{\mathrm{ns}}$ \\
\hline $\begin{array}{l}\text { Irrigation } \times \text { growth stage } \\
\times \text { concentration }\end{array}$ & 4 & $193.8440606^{* *}$ & $62698.164^{* *}$ & $47961.0313^{* *}$ & $113982329^{* *}$ & $6.4478125^{* *}$ & $3104268.13^{* *}$ & $6225254.8^{\mathrm{ns}}$ & $68.0248325^{* *}$ & $1.1266919^{* *}$ & $5.862063^{* *}$ \\
\hline Error & 48 & 13.779679 & 6033.332 & 3643.335 & 5335882 & 0.4183125 & 156055.86 & 4067289 & 3.995112 & 0.1989550 & 1.392625 \\
\hline C.V & & 6.14 & 9.25 & 9.64 & 8.48 & 1.82 & 8.63 & 10.12 & 8.21 & 4.44 & 1.73 \\
\hline
\end{tabular}

${ }^{*},{ }^{* *}$ and ns: significant at $0.05,0.01$ probability level and no significant, respectively.

Tab. 2. Individual effects of irrigation, growth stages and titanium concentrations on some agronomic traits, gluten and starch of wheat

\begin{tabular}{|c|c|c|c|c|c|c|c|c|c|c|}
\hline Treatments & $\begin{array}{c}\text { Plant } \\
\text { height }(\mathrm{cm})\end{array}$ & $\begin{array}{c}\text { Ear weight } \\
\left(\mathrm{g} \cdot \mathrm{m}^{-2}\right)\end{array}$ & Ear number & Seed number & $\begin{array}{l}\text { 1000-seed } \\
\text { weight }(\mathrm{g})\end{array}$ & $\begin{array}{c}\text { Final yield } \\
\left(\mathrm{kg} \cdot \mathrm{ha}^{-1}\right)\end{array}$ & $\begin{array}{l}\text { Biomass } \\
\left(\mathrm{kg}^{2} \cdot \mathrm{ha}^{-1}\right)\end{array}$ & $\begin{array}{c}\text { Harvest } \\
\text { index }(\%)\end{array}$ & Gluten(\%) & Starch(\%) \\
\hline \multicolumn{11}{|l|}{ Irrigation } \\
\hline Normal irrigation & $61.9093 a$ & $841.48 \mathrm{a}$ & $657.08 \mathrm{a}$ & $28796 a$ & $37.7725 a$ & $4826.2 \mathrm{a}$ & $20602.3 a$ & $26.8138 \mathrm{a}$ & $11.77825 \mathrm{a}$ & $72.3400 \mathrm{a}$ \\
\hline Water deficit stress & $58.8378 \mathrm{~b}$ & $836.42 a$ & $594.15 \mathrm{~b}$ & $25711 b$ & $32.9775 b$ & $4321.8 \mathrm{~b}$ & $19244.0 \mathrm{~b}$ & $21.8743 \mathrm{~b}$ & $8.27975 b$ & $63.7225 b$ \\
\hline \multicolumn{11}{|l|}{ Growth stages } \\
\hline Stem elongation & $59.993 a$ & $851.46 \mathrm{a}$ & $627.95 a$ & $27017.6 a$ & $35.4725 a$ & $4678.38 \mathrm{a}$ & $19146.2 \mathrm{a}$ & $25.2730 \mathrm{a}$ & $10.00300 \mathrm{a}$ & $68.0825 a$ \\
\hline Flowering & $60.754 a$ & $826.44 a$ & $623.28 \mathrm{a}$ & $27462.0 \mathrm{a}$ & $35.2275 \mathrm{a}$ & $4469.63 b$ & $20700.0 \mathrm{a}$ & $23.4150 \mathrm{~b}$ & $10.05500 \mathrm{a}$ & $67.9800 \mathrm{a}$ \\
\hline \multicolumn{11}{|l|}{ Titanium } \\
\hline Titanium dioxide 0.01 & $58.581 \mathrm{~b}$ & $831.18 \mathrm{~b}$ & $629.44 \mathrm{~b}$ & $27103.6 \mathrm{~b}$ & $35.6625 a$ & $4538.8 \mathrm{~b}$ & $19838.4 \mathrm{bc}$ & $25.4119 b$ & $10.3406 a$ & $68.7313 \mathrm{a}$ \\
\hline Titanium dioxide 0.02 & $59.973 \mathrm{ab}$ & $899.93 a$ & $675.31 \mathrm{a}$ & $30145.6 \mathrm{a}$ & $35.7375 a$ & $4896.1 \mathrm{a}$ & $21153.9 \mathrm{ab}$ & $28.3394 a$ & $10.2350 \mathrm{ab}$ & $68.5875 a$ \\
\hline Titanium dioxide 0.03 & $59.789 \mathrm{ab}$ & $836.51 \mathrm{~b}$ & $625.75 b$ & $27460.7 b$ & $35.3438 \mathrm{ab}$ & $4670.2 \mathrm{ab}$ & $17782.4 \mathrm{c}$ & $23.6744 \mathrm{c}$ & $9.9438 \mathrm{bc}$ & $68.3250 \mathrm{ab}$ \\
\hline Bulk titanium & $61.733 a$ & $866.14 a b$ & $628.50 \mathrm{~b}$ & $27249.5 b$ & $35.0750 \mathrm{~b}$ & $4670.0 \mathrm{ab}$ & $21481.4 a$ & $22.1575 d$ & $9.8819 \mathrm{c}$ & $67.5563 b c$ \\
\hline Control & $61.792 \mathrm{a}$ & $760.98 \mathrm{c}$ & $569.06 \mathrm{c}$ & $24239.7 \mathrm{c}$ & $35.0563 b$ & $4094.8 \mathrm{c}$ & $17782.4 \mathrm{~d}$ & $22.1369 \mathrm{~d}$ & $9.7438 \mathrm{c}$ & $66.9563 \mathrm{c}$ \\
\hline
\end{tabular}

Values within the each column and followed by the same letter are not different at $p \leq 0.05$ by an ANOVA protected Duncan’s Multiple Range Test 
Tab. 3. Combined effects of irrigation, growth stages and titanium concentrations on some agronomic traits, gluten and starch of wheat

\begin{tabular}{|c|c|c|c|c|c|c|c|c|c|c|c|c|}
\hline Irrigation & Growth stages & Titanium & $\begin{array}{c}\text { Plant } \\
\text { height }(\mathrm{cm})\end{array}$ & $\begin{array}{c}\text { Ear weight } \\
\left(\mathrm{g} \cdot \mathrm{m}^{-2}\right)\end{array}$ & Ear number & $\begin{array}{c}\text { Seed } \\
\text { number }\end{array}$ & $\begin{array}{l}\text { 1000-seed } \\
\text { weight }(\mathrm{g})\end{array}$ & $\begin{array}{c}\text { Final yield } \\
\left(\mathrm{kg}^{\left.-\mathrm{ha}^{-1}\right)}\right.\end{array}$ & $\begin{array}{l}\text { Biomass } \\
\left(\mathrm{kg} \mathrm{ha}^{-1}\right)\end{array}$ & $\begin{array}{c}\text { Harvest } \\
\text { index }(\%)\end{array}$ & Gluten(\%) & Starch(\%) \\
\hline \multirow{10}{*}{ 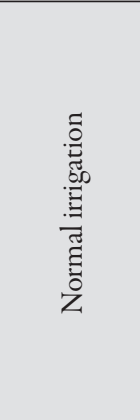 } & \multirow{5}{*}{ 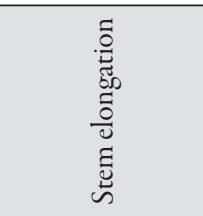 } & Titanium dioxide 0.01 & $60.36 \mathrm{bcd}$ & 883.7 def & $647.3 \mathrm{cdef}$ & 27820 bcdef & $37.35 \mathrm{~d}$ & $4986 \mathrm{~cd}$ & $20100 \mathrm{de}$ & $26.34 \mathrm{bc}$ & $12.29 \mathrm{a}$ & $73.95 \mathrm{a}$ \\
\hline & & Titanium dioxide 0.02 & $59.48 \mathrm{cde}$ & $1226.0 \mathrm{a}$ & $990.3 \mathrm{a}$ & $43900 \mathrm{a}$ & $38.65 \mathrm{bc}$ & $6576 \mathrm{a}$ & $20800 \mathrm{de}$ & $38.28 \mathrm{a}$ & $12.04 \mathrm{a}$ & $71.28 \mathrm{e}$ \\
\hline & & Titanium dioxide 0.03 & $57.91 \mathrm{def}$ & $1037.4 \mathrm{bc}$ & $706.8 \mathrm{~cd}$ & $30810 \mathrm{bc}$ & $38.05 \mathrm{bdc}$ & $5684 b$ & $1886 \mathrm{~g}$ & $27.10 \mathrm{~b}$ & $11.92 \mathrm{ab}$ & $71.47 \mathrm{de}$ \\
\hline & & Bulk titanium & $65.80 \mathrm{ab}$ & 763.3 fghi & $452.3 \mathrm{ij}$ & $18880 \mathrm{j}$ & $37.05 \mathrm{de}$ & $4193 \mathrm{fgh}$ & $16770 \mathrm{f}$ & $25.23 \mathrm{bcd}$ & $11.34 \mathrm{c}$ & 72.00 cde \\
\hline & & Control & $59.71 \mathrm{~cd}$ & $674.1 \mathrm{hi}$ & $718.3 c$ & $18810 j$ & $38.25 \mathrm{bcd}$ & $3760 \mathrm{hi}$ & $15980 \mathrm{f}$ & $26.57 b c$ & $11.18 \mathrm{c}$ & $72.93 \mathrm{abc}$ \\
\hline & \multirow{5}{*}{$\begin{array}{l}\stackrel{b}{0} \\
\text { 离 } \\
\text { 总 } \\
\end{array}$} & Titanium dioxide 0.01 & $64.48 \mathrm{abc}$ & $909.6 \mathrm{cdef}$ & $535.3 \mathrm{ghi}$ & 22380 ghij & $39.00 \mathrm{~b}$ & 3895 ghi & $16790 \mathrm{f}$ & $27.56 \mathrm{~b}$ & $12.10 \mathrm{a}$ & $71.38 \mathrm{e}$ \\
\hline & & Titanium dioxide 0.02 & 58.92 cde & 727.3 ghi & $477.3 \mathrm{hij}$ & $20380 \mathrm{ij}$ & $40.78 \mathrm{a}$ & $4246 \mathrm{fgh}$ & $16740 \mathrm{f}$ & $25.67 \mathrm{bc}$ & $12.38 \mathrm{a}$ & 72.25 bcde \\
\hline & & Titanium dioxide 0.03 & $63.07 \mathrm{abcd}$ & 718.0 ghi & $570.0 \mathrm{fgh}$ & 24870 efgh & $37.60 \mathrm{~cd}$ & 4102 fghi & $17060 \mathrm{f}$ & $27.14 b$ & $11.94 \mathrm{ab}$ & $71.75 \mathrm{cde}$ \\
\hline & & Bulk titanium & $60.36 \mathrm{bcd}$ & $658.6 \mathrm{i}$ & $895.8 \mathrm{~b}$ & $40710 \mathrm{a}$ & $35.05 \mathrm{gh}$ & $6384 \mathrm{a}$ & $28610 a$ & 22.73 def & $11.44 \mathrm{bc}$ & $73.58 \mathrm{ab}$ \\
\hline & & Control & $68.50 \mathrm{a}$ & 785.0 efghi & $605.5 \mathrm{defg}$ & 25810defg & $35.95 \mathrm{ef}$ & 4430efg & $20690 \mathrm{de}$ & $21.53 \mathrm{ef}$ & $11.17 \mathrm{c}$ & $72.83 \mathrm{abcd}$ \\
\hline \multirow{10}{*}{ 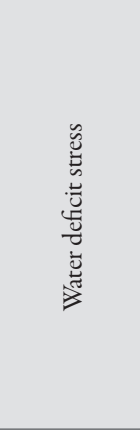 } & \multirow{5}{*}{ 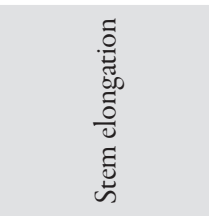 } & Titanium dioxide 0.01 & $59.92 \mathrm{~cd}$ & 931.5 cde & $690.3 \mathrm{cde}$ & $29810 \mathrm{bcd}$ & $34.00 \mathrm{gh}$ & 4951cde & $22110 \mathrm{~cd}$ & $22.45 \mathrm{def}$ & $8.760 \mathrm{ef}$ & $64.58 \mathrm{~g}$ \\
\hline & & Titanium dioxide 0.02 & $60.13 \mathrm{~cd}$ & $1105.5 \mathrm{ab}$ & 619.3cdefg & 26980cdef & $34.68 \mathrm{gh}$ & $5327 \mathrm{bc}$ & $25180 \mathrm{~b}$ & $21.30 \mathrm{ef}$ & $9.375 \mathrm{~d}$ & $66.30 \mathrm{f}$ \\
\hline & & Titanium dioxide 0.03 & $64.08 \mathrm{abc}$ & 813.8 defghi & $649.0 \mathrm{cdef}$ & 28480 bcde & $32.23 \mathrm{j}$ & 3968 ghi & 18500 ef & $20.99 \mathrm{f}$ & 7.925 hij & $61.68 \mathrm{i}$ \\
\hline & & Bulk titanium & $53.37 \mathrm{fg}$ & $688.8 \mathrm{hi}$ & $572.0 \mathrm{fgh}$ & 23830 fghi & $33.50 \mathrm{hi}$ & 3969 ghi & $16350 \mathrm{f}$ & $24.12 \mathrm{cde}$ & $7.123 \mathrm{j}$ & $62.78 \mathrm{hi}$ \\
\hline & & Control & $58.83 \mathrm{cde}$ & $675.2 \mathrm{hi}$ & 520.3 ghij & 20870 hij & $31.35 \mathrm{jk}$ & $3555 \mathrm{i}$ & $16790 \mathrm{f}$ & $20.36 \mathrm{f}$ & $7.665 \mathrm{ij}$ & $63.88 \mathrm{gh}$ \\
\hline & \multirow{5}{*}{$\begin{array}{l}b_{0}^{0} \\
\stackrel{\Xi}{0} \\
\frac{\partial}{0}\end{array}$} & Titanium dioxide 0.01 & $49.57 \mathrm{~g}$ & $850.9 \mathrm{dfeg}$ & $645.0 \mathrm{cdef}$ & 28410 bcde & $32.30 \mathrm{ij}$ & $4323 \mathrm{fgh}$ & $20360 \mathrm{de}$ & $25.13 \mathrm{bcd}$ & $8.975 \mathrm{de}$ & $65.03 \mathrm{~g}$ \\
\hline & & Titanium dioxide 0.02 & $61.01 \mathrm{bcd}$ & 825.7 defgh & $614.5 \mathrm{cdefg}$ & $29330 \mathrm{bcd}$ & $32.55 \mathrm{ij}$ & $4322 \mathrm{fgh}$ & $21890 \mathrm{~cd}$ & $20.63 \mathrm{f}$ & $8.470 \mathrm{efg}$ & $64.53 \mathrm{~g}$ \\
\hline & & Titanium dioxide 0.03 & $54.10 \mathrm{efg}$ & 776.9 fghi & $577.3 \mathrm{fgh}$ & 25960 defg & 33.50 hi & $4225 \mathrm{fgh}$ & $16680 \mathrm{f}$ & $26.95 \mathrm{bc}$ & $8.400 \mathrm{fgh}$ & $62.93 \mathrm{hi}$ \\
\hline & & Bulk titanium & $66.90 \mathrm{a}$ & 786.4 efghi & $594.0 \mathrm{efg}$ & 25580 defg & $34.70 \mathrm{gh}$ & 3941 ghi & $24200 \mathrm{bc}$ & $16.47 \mathrm{~g}$ & $7.590 \mathrm{ij}$ & $61.88 \mathrm{i}$ \\
\hline & & Control & $60.13 \mathrm{~cd}$ & $941.3 \mathrm{~cd}$ & $432.3 \mathrm{j}$ & $31800 \mathrm{~b}$ & $30.98 \mathrm{k}$ & $4634 \mathrm{def}$ & $23970 \mathrm{bc}$ & $20.18 \mathrm{f}$ & 8.075 ghi & $63.68 \mathrm{gh}$ \\
\hline
\end{tabular}

Values within the each column and followed by the same letter are not different at $p \leq 0.05$ by an ANOVA protected Duncan's Multiple Range Test. 
206

biomass. Water deficit stress decreased biomass regardless of the growing stage (Tab. 2). Moreover, titanium dioxide nanoparticles $(0.02 \%)$ and titanium oxide (bulk) application produced the highest biomass (Tab. 2). The highest biomass was obtained when non-stressed plants were treated with titanium oxide (bulk) during the flowering stage (Tab. 3). This is in agreement with Zheng et al. (2005), who reported that the significant effect of titanium nanoparticles on spinach is probably attributed to the small particle size, which allows its penetration into the seed during the treatment period. It seems that bulk titanium could not penetrate into the plants; therefore, the results were not as marked as those of the treatment with nanoparticles. Increase of growth and yield may be due to the positive effects of titanium in different cellular mechanisms. For instance, improvement of photosynthesis and increase in chlorophyll content are two possible reasons for this. Owolade et al. (2008) reported that the seed yield of cowpea (Vigna unguiculata Walp) was increased when treated (as foliar application) with nano-sized titanium dioxide. They concluded that it may be due to the photocatalyst ability of the nano-sized titanium dioxide, which leads to an increased photosynthetic rate. Similar yield increases were reported in rice with a corresponding reduction in the incidence Curvularia leaf spot and bacteria leaf blight disease (Chao and Choi, 2005). Harvest index was affected by all individual and combined effects (Tab. 1). Water deficit stress decreased harvest index especially at the flowering stage (Tab. 2). Titanium dioxide nanoparticles $(0.02 \%)$ caused the maximum harvest index, whereas titanium oxide (bulk) and control treatments were related to the minimum (Tab. 2). When the effects of the experimental factors were combined, the maximum harvest index was related to non-stressed plants treated with titanium dioxide nanoparticles $(0.02 \%)$ at the flowering stage (Tab. 3).

Irrigation withholding and titanium application had a significant effect on gluten content but the growing stage had no significant effect on this trait (Tab. 1). It is worth mentioning that the combined effect of irrigation, growing stages, and titanium concentrations was significant as well (Tab. 1). A drastic decrease in 1000 seed weight was recorded. This result is in agreement with those of Khan et al. (2005) and Qadir et al. (1999), who observed that 1000 seed weight of wheat was reduced due to water stress. It might be concluded from the results of this study that water deficit stress caused a significant reduction in vegetative growth, yield, and yield components of wheat. We have suggested in our recent work that titanium increases wheat growth, yield and yield components and improves gluten and starch content of the seeds. Among the different titanium treatments, titanium dioxide nanoparticles at $0.02 \%$ concentration produced the highest yield and yield components and improved gluten and starch content, especially under conditions of complete irrigation. Zheng et al. (2005) demonstrated that titanium nanoparticles helped the water absorption by the spinach and improved growth. So, their results showed that the growth of spinach plants greatly improved at 250-4,000 ppm nano titanium dioxide concentrations, but there was no improvement at higher concentrations. Similarly, we have found that when titanium concentration was more than $0.02 \%$, plant growth and production would be decreased. This finding shows that the positive effect of titanium depends on its concentration. In addition, particle size plays an important role in absorption by plants. Because titanium oxide (bulk) has greater size than titanium dioxide nanoparticles, it could not be absorbed by plants as easily as the nanoparticles

Gluten decreased due to water deficit stress and increased on account of titanium dioxide nanoparticles application (Tab.2). The highest gluten content was observed when non-stressed plants were treated with titanium dioxide nanoparticles either during the stem elongation stage or during the flowering stage (Tab. 3). Starch content was affected and decreased because of water deficit stress (Tab. 1 and 2). There was no significant difference between the growing stages in terms of starch content (Tab. 2). Titanium application increased starch content in comparison with control treatment (Tab.2). The highest starch content was obtained when non-stressed plants were treated with titanium dioxide nanoparticles $(0.01 \%)$ during the stem elongation stage (Tab. 3). Gluten and starch content were affected by water deficit stress so that water deficit stress caused a significant decrease in these qualitative traits. Zhao et al. (2009) demonstrated that protein components are very sensitive to drought stress. An increase of gluten and starch in treated plants with titanium may be due to the positive correlation between titanium application and photosynthesis rate so that titanium application improved $\mathrm{CO}_{2}$ metabolism in spinach. This increase in photosynthesis was attributed to an improvement of rubisco activity (Gao et al., 2006).

\section{References}

Carvajal MF, Martínez-Sánchez F, Alcaraz CF (1994). Effect of Ti on some physiological activity indicators of Capsicum anuum L. plants. J Hortic Sci 69:427-432.

Chao SHL, Choi HS (2005). Method for providing enhanced photosynthesis. Korea Research Institute of Chemical Technology, Jeju, South Korea, 10 p.

Dumon JC, Ernst WHO (1988). Titanium in plants. J Plant Physiol 133:203-209.

Fulai L, Christian R, Mathias J (2004). Drought stress effect on carbohydrate concentration during early reproductive development. Field Crops Res 86:1-13.

Gao F, Hong F, Liu C, Zheng L, Su M, Wu X, Yang F, Wu C, Yang $\mathrm{P}$ (2006). Mechanism of nano-anatase $\mathrm{TiO}_{2}$ on promoting photosynthetic carbon reaction of spinach. Biol Trace Element Res 11(3):239-254.

Giménez JL, Martínez-Sánchez F, Moreno A (1990). Effect of 
Ti on yield of Capsicum anuum, L., 123-128 p. In : Proc. of III Symposium Nacional de Nutrición Mineral de las Plantas, SPIC-UIB, eds., Nutrición Mineral bajo condiciónes de Estrés.

Graveland A, Henderson MH (2000). Composition and standard of gluten proteins, 90-99 p. In: Schfirld JD (Ed.). Wheat stucure, Biochemistry and functionality. Royal Soc of Chem Cambridge.

Grenda A (2003). Tytanit-an activator of metabolic processes. Chemicals in Sust Agric 4:263-269.

Hruby M, Cigler P, Kuzel S (2002). Titanium in plant nutrition: The contribution to understanding the mechanism of titanium action in plants. J Plant Nutr 25:577-598.

Inamullah ZA, Swati A, Siraj D (1999). Evaluation of lines for drought tolerance in heat (Triticum aestivum L.). Sci Khy J 12(2):39-48.

Khanzada S, Ala A, Yasin Ashraf M, Shirazi MU, Alam SM, Ansari R, Ali M, Mujataba SM, Khan MA (2001). Effect of water stress on yield and yield components of different Guar (Cyamopsis tetragonaloba L.) genotypes. Pak J Bio Sci 4:371-374.

Lu CM, Zhang CYW, Tao MX (2002). Research of the effect of nanometer on germination and growth enhancement of Glycine max L. and its mechanism. Soybean Sci 21:168-172.

Malik MA, Hassan F(2002). Response of wheat genotypes on suppression of weeds under rainfed conditions. Pak J Agri Eng Vet Sci 18(2):18-22.

Navarro E, Baun A, Behra R, Hartmann NB, Filser J, Miao A, Quigg A, Santschi PH, Sigg L (2008). Environmental behavior and ecotoxicity of engineered nanoparticles to algae, plants, and fungi. Ecotoxicology 17:372-386.
Owolade OF, Ogunleti DO, Adenekan MO (2008). Titanium dioxide affected diseases, development and yield of edible cowpea. Electronic J Environ Agric Food Chem 7(5):29422947.

Pais I (1983). The biological importance of titanium. J Plant Nutr 6:3-131.

Qadir G, Saeed M, Mumtaz C (1999). Effect of water stress on growth and yield performance of four wheat cultivars. Pak J Biol Sci 2(1):236-239.

Ruffini Castiglione M, Cremonini R (2009). Nanoparticles and higher plants. Caryologia 62:161-165.

SAS Institute Inc., 2002. The SAS System for Windows, Release 9.0. Statistical Analysis Systems Institute, Cary, NC, USA.

Tompkins DK, Fowler DB, Wright AT (1991). Water use by no tillage winter wheat influence of seed rate and row spacing. Agron J 766-769.

Yaghoubi S, Schwietert CW, Mc Cue JP (2000). Biological roles of titanium. Biol Trace Element Res 78:205-217.

Zhao CX, He MR, Wang ZL, Wang YF, Lin Q (2009). Effects of different water availability at post-anthesis stage on grain nutrition and quality in strong-gluten winter wheat. Com Ren Biol 332: 759-764.

Zheng L, Hong F, Lu S, Liu C (2005). Effect of nano-TiO ${ }_{2}$ on strength of naturally aged seeds and growth of spinach. Biol Trace Elem Res 105:83-91. 